\title{
Pooled Dissolution Testing: A Primer
}

\section{Royal Hanson* \\ Hanson Researcb Corp.}

Chatswortb, CA

Dr: Lee T. Grady of USP bas proposed a new labor-saving method for dissolution testing. The proposal calls for testing of pooled samples, thereby reducing the analytical burden. This article reviews the principles bebind the USP proposal, as well as two practical methods for achieving pooled dissolution testing.

Lee T. Grady, director of the Drug Standards Division of the United States Pharmacopeia (USP), has proposed a new concept in the field of dissolution testing: pooled dissolution (1). A key principle in this proposal is the "batch characteristic" of dissolution testing, and a primary benefit is the "reduction of the analytical burden." USP has demonstrated itself to be a forwardlooking, world-class organization by taking the lead in such areas as the international barmonization of standards and the environmental impact of modern analytical requirements for reagents (2). Significantly, this new proposal is bistoric in the
Robert J. Jarnutowski

InPharm Tech

Stephenson, $M I$ field of dissolution testing. For the first time in 25 years, a fundamental paradigm shift is being offered in an analytical technique - and it provides not for an increase, but a reduction of the current dissolution work load.At its first level, pooled dissolution refers to assaying a pooled sample from individual dissolution vessels, simplifying the current task of multiple analyses from individual vessels, and thus providing some relief to the analytical burden. At its second level, pooled dissolution refers to the testing of multiple units in a single vessel, achieving still greater work load savings for the modern dissolution lab. It is also suggested that a new apparatus for testing multiple units in a single vessel may be considered. This article provides an overview of the new USP proposal, along with two practical methods for pooled dissolution testing that are attainable with commercial equipment available today.

\section{The New USP Proposal}

Figure 1 illustrates the current dissolution method in relation to the new USP proposal for pooled testing and analyses. Traditional dissolution requires individual samples to be analyzed from individual vessels. Level 1 pooled
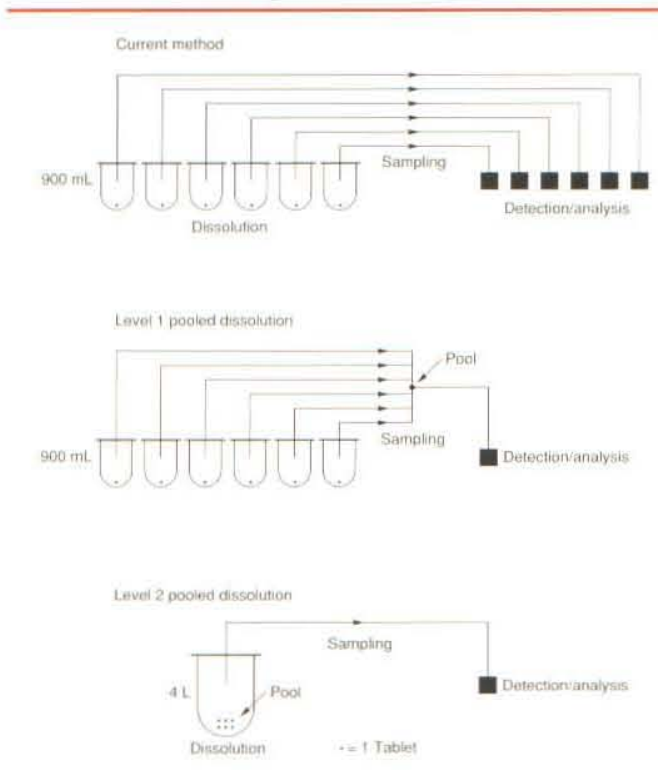

Figure 1: Pooled Dissolution Technique dissolution proposes assaying pooled specimens from individual dissolution vessels, and level 2 proposes that multiple units (of soluble drugs) be tested in a single vessel.

When evaluated from a systems eng ineering approach, it is apparent that pooled dissolution sim plifies (through consolidation) the task of sample analysis. With level 1 test- ing, individual dissolution specimen assay is reduced by a factor of six. Furthermore, with level 2 testing, equipment setup requirements are similarly reduced.

In his proposal, Grady comments that two primary USP objectives are to ensure "fitness for use" of pharmaceutical products and to establish analytical guidelines to minimize or prevent any actual bioavailability problems in the treatment of patients. He points out that in the past 25 years, not a single problem report "stood or fell on the basis of any single dosage unit," but rather always on the basis of batch phenomena. That is, in terms of product efficacy, problems in batch-to-batch and product-to-product differences have been found to be significant, whereas unit-to-unit differences have not. The proposal for pooled dissolution testing is therefore a scientific recognition of the batch characteristic of dissolution testing and a logical conclusion based on this historical experience.

It is noted that certain theoretical and simulated statistical models may suggest that pooled dissolution could result in increased "stage 2 " acceptance testing $(3,4)$. However, with the 70 USP First Case products (that is, high solubility, rapidly dissolving, $75 \%$ in $45 \mathrm{~min}$ ) that are proposed for pooled dissolution testing, this should not be a problem $(5,6)$. 


\section{Pooled Dissolution Testing....continued}

\section{Level 1 Pooled Dissolution Technique}

Commercially available equipment for level 1 pooled dissolution testing includes the SR8-Plus dissolution test station and Dissoette

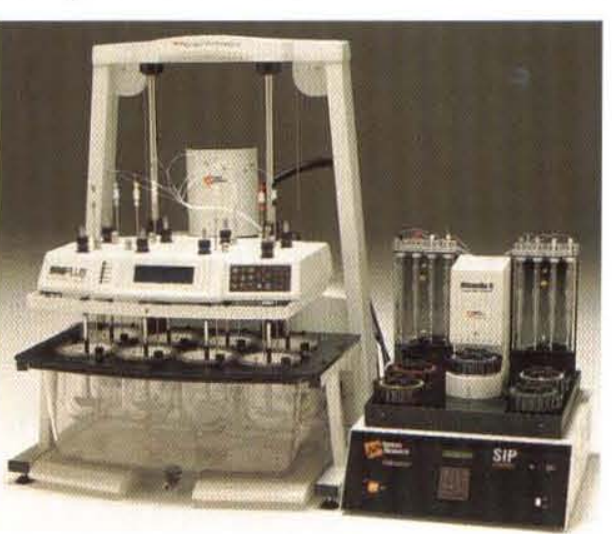

Figure 2: Hanson Dissolution Test Station and Autosampler autosampler ( $\mathrm{H}$ a $\mathrm{n}$ s o n Research Corp., Chatsworth, CA), shown in Figure 2, and the Alliance highperformance liquid chromatography (HPLC)system (Waters Corp., Milford, MA), shown in Figure 3. For example, the Hanson dissolution test station and autosampler provide a platform for standard dissolution testing, including automated test protocol, sampling, and collection into Alliance HPLC carousels. Groups of six samples (typically) are automatically archived in coded carousels for later analysis. As many as 19 such sample groups may then be analyzed without manual intervention.

Using modern

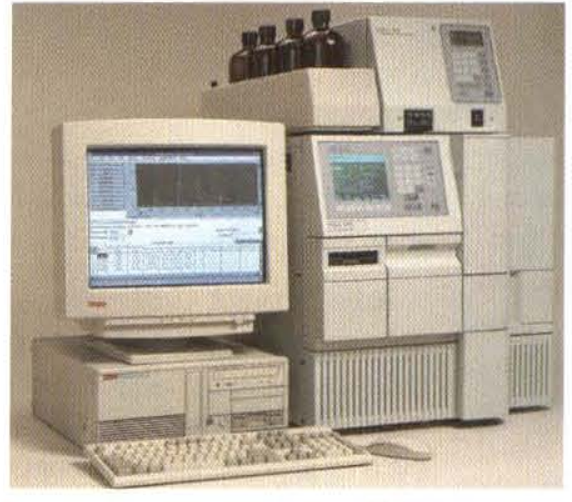

Figure 3: Waters Alliance HPLC System
HPLC instrumentation, it is possible to analyze pooled dissolution samples collected in the normal manner, as described above, using the Dissoette autosampler. The analysis of pooled samples is accomplished by allowing the HPLC system to automatically pool the samples as part of an auto-addition injection routine. Separate, discrete samples are obtained from the dissolution bath and placed into the sample-handling compartment of the HPLC separations module. Co-injections using the auto-addition function can then be made corresponding to the number of vessels (as many as 10) used in the dissolution test.

The results of one such experiment using an HPLC

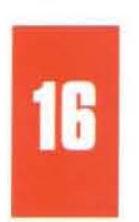
method for the analysis of prednisone calibrator tablets are shown in Table I. Samples
Sample:

Dissolution Test: Automated Pooling:

\begin{tabular}{|c|c|c|c|c|c|}
\hline $\begin{array}{l}\text { Dissoltuion } \\
\text { Sample } \\
\text { Time }\end{array}$ & $\begin{array}{l}\text { Ind. Assay } \\
\text { Calculated } \\
\text { Avg. }\end{array}$ & $\begin{array}{c}\text { Man. Pool } \\
\text { Amount }\end{array}$ & $\%$ Error & $\begin{array}{l}\text { Auto Pool } \\
\text { Amount }\end{array}$ & $\%$ Error \\
\hline $\begin{array}{l}10 \\
30 \\
60\end{array}$ & $\begin{array}{l}10.81 \\
20.43 \\
28.69\end{array}$ & $\begin{array}{l}10.78 \\
20.38 \\
28.59\end{array}$ & $\begin{array}{l}0.3 \\
0.2 \\
0.3\end{array}$ & $\begin{array}{l}10.76 \\
20.30 \\
28.45\end{array}$ & $\begin{array}{l}0.5 \\
0.6 \\
0.8\end{array}$ \\
\hline
\end{tabular}

were first analyzed in the normal fashion by quantitating six separate samples. These results were then compared with both a manually pooled sample performed by an analyst with a pipette, and an automated pooled sample performed using the auto-addition routine of the Alliance HPLC system.

As shown in Table I, these results agree within experimental error, indicating that the HPLC instrument itself can be used to pool the samples in an automated fashion. Not only does the automatic pooling reduce the possibility for operator error, it also has the advantage of allowing reanalysis of any sample (both pooled and individually, if necessary). Additional features of the system also allow sample transfers, if neutralization is required, and mixing for reagent addition.

\section{Level 2 Pooled Dissolution Technique}

A 4-L dissolution test system, such as that shown in Figure 4, would be appropriate for the multiple-unit approach. The Hanson 4-L test system shown has been in commercial and scientific use for 10 years, including work on low-solubility drugs (for example, thalidomide studies) at FDA's National Center for Drug Analysis in St. Louis, Missouri (7). Moreover, if desired, as many as three 4-L flasks can be accommodated with ease in one bath. The analyst can significantly improve the throughput of a single bath that is equipped with three vessels. For example, if 6 units were to be tested in each vessel, only 3 measurements would have to be made compared to 18 indi-

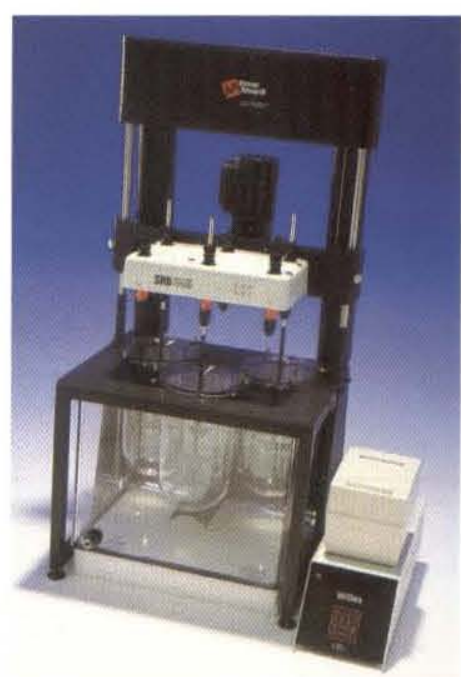

Figure 4: Hanson 4-Liter Dissolution Test Station vidual measurements - a sixfold improvement in measurement efficiency.

In terms of spectrophotometric measurement, the six samples in 4 $\mathrm{L}$ of media will yield a more concentrated solution by a factor of 1.35 (6 units $\times 900 \mathrm{~mL}=5400 \mathrm{~mL} ; 5400 \mathrm{~mL} / 4000 \mathrm{~mL}=1.35$ ). In most cases, a modern spectrophotometer will have a sufficiently dynamic measuring range to accommodate this increased absorbance and will provide valid concentra-
tion values. However, the analyst should be prepared to change the measurement cell's pathlength if the additional sample raises the solution's absorbance outside the range for optimum accuracy (usually 0.4 to 1.4 absorbance units).
Standard USP conditions, six vessel test with HPLC analysis Auto-Addition feature of Waters Alliance HPLC System 
If one is using a spectrophotometer that samples automatically from six vessels, three of the sample cells could be used for the first threevessel bath while the other three sample cells could be used for a second bath equipped with three additional 4-L vessels.

Efficiency is not only improved in the measurement process, but in other areas as well. Using this system, only 3 rather than 18 vessels need to be installed, aligned, and validated. Only 3 rather than 18 vessels need to be emptied, washed, reinstalled, and filled again with media after each run. Bench space requirements are reduced by a factor of three. If two baths each equipped with three 4-L vessels are used, then results can be obtained for 36 units in the bench space currently required for testing 12 units, because the same detector can be used for pooled sample determinations on all 36 units.

In these vessels, an extra-long (21-in.) USP paddle is used, with a media volume of $4000 \mathrm{~mL}$. For readily soluble units, the rate of dissolution will likely change little compared to the $5400 \mathrm{~mL}$ total volume one would use if six separate vessels were used. The USPSubcommittee on Dissolution and Bioavailability is willing to consider validated data that demonstrate that multiple units (of soluble drugs) can be tested in a single vessel with no significant change in the mean observed amount dissolved relative to that obtained by testing individual units.

\section{Conclusions}

Grady (1) has suggested that a reasonable goal for those who perform analytical testing on articles defined in USP would be to consider means to reduce the cost of this testing with no loss of the fundamental assurance that the article is still suitable for its intended use. Dissolution testing is an obvious area to address because of the great analytical burden implicit in the current methods used. The pooling of samples is a way to help reduce the number of determinations that are made currently to evaluate articles. In fact, the first 70 monographs for pooled samples are scheduled to appear in Supplement 8 (May 1998) to USP.

Recall that dissolution testing is indeed a batch characteristic of the article being examined in spite of the fact that USP at present typically requires the utilization and testing of six individual articles at the first stage. Grady's paper suggested reducing the analytical burden by performing the assay on pooled specimens from individual vessels rather than the current practice of multiple analyses from individual vessels. This reduces the analytical measurement burden by a factor of six.

However, this does little to address the other tasks, which are rather time-consuming and labor-intensive, that are associated with the setup and completion of a dissolution run. A second approach offered by Grady was to consider multiple units in a single vessel, including consideration of a new design that would support the testing of multiple units in a single vessel. Commercial equipment is available today to achieve both levels of pooled dissolution testing.

\section{References}

1. L. Grady, "Call for a Third Generation of Dissolution Testing and Principles of Application - An Initial Initiative Based on Recognition of Dissolution as a Batch Characteristic," Pharm. Forum 21 (1), 177 (1995).

2. USP Open Conference, Current Revision Issues - Analytical Methods \& International Harmonization, 21-24 May 1995, Chicago, IL.

3. C. Wojcik et al., "An Evaluation of the Dissolution Test Acceptance Sampling Procedure for Pooled Samples," Pharm. Forum 21 (4), 1169 (1995).

4. "Pooled Sampling - Comments Received," Pharm. Forum 23 (3), 4243 (1997).

5. "In-Process Revision - Monographs," Pharm. Forum 22 (5), 2777 (1996).

6. "Pooled Sampling - Response to Comments Received," Pharm. Forum 23 (3), 4248 (1997).

7. D. Cox, Senior Scientist, FDA, St. Louis, MO, private communication, 25 August 1997.

Reprinted courtesy of Pbarmaceutical Tecbnology, An Advanstar Publication. Reference Pharmaceutical Tecbnology 1997 Tableting and Granulation Yearbook for original publication.

Royal Hanson is president and CEO of Hanson Research Corporation, 9810 Variel Avenue, Chatsworth, CA 91311, tel. (818) 882-7266, fax (818) 882-9470. Michael E. Swartz, PhD, is senior scientist at Waters Corporation, Milford, MA. Robert J. Jarnutowski is president of InPharm Tech, Stephenson, MI. 